\title{
EL EFECTO AGUDO DEL EJERCICIO EN LA PRESIÓN INTRAOCULAR:
}

\author{
META-ANÁLISIS
}

\author{
Carolina Alemán ${ }^{1}$ y Andrea Solera-Herrera ${ }^{2}$
}

(1) Posgrado en Ciencias del Movimiento Humano, Universidad Nacional;

Docente, Instituto Hellen Keller, Costa Rica

(2) Escuela de Educación Física y Deportes y Centro de Investigación en

Ciencias del Movimiento Humano, Universidad de Costa Rica

Envío original: 2018-09-28. Reenviado: 2018-12-17, 2019-01-04. Aceptado: 2019-03-06. Publicado: 2019-04-10.

\section{Resumen}

La presión intraocular (PIO) alta afecta la salud visual, principalmente es un factor de riesgo para el glaucoma. El objetivo de este estudio, fue conocer los efectos del ejercicio agudo sobre la PIO. Metodología: se Meta-analizaron estudios experimentales que evaluaron, el efecto de la actividad física aguda sobre PIO. La búsqueda de datos abarco nueve bases de datos multidisciplinarias. Se aplicó un protocolo PRISMA, con criterios de elegibilidad, calidad metodológicas, y extracción de la información por dos investigadores para garantizar reproductibilidad. El meta-análisis fue de efectos aleatorios, heterogeneidad con DerSimonian-Lairds. Resultados: Se analizaron veintinueve artículos para obtener los tamaños de efecto (TE), los grupos experimentales incluyeron a 1016 personas y los grupos control 120 personas. Un total de $102 \mathrm{TE}$ delta fueron calculados. Los resultados de este estudio muestran para el grupo control un tamaño de efecto de 0,31 (IC $95 \%=$ de -0.46 a $\left.1.08, Q=16.15, \mathrm{I}^{2}=81.42 \mathrm{p}=\leq 0,05\right)$. Para los grupos experimentales se obtuvo un efecto de $-0,68$ (IC95\%= de -0.95 a $-0.41, Q=1573.49, I^{2}=$ 99.80, $\mathrm{p}=\leq 0,05)$. En el ejercicio aeróbico agudo el TE fue de $-1,74$ (IC95\%= de $-2.11 \mathrm{a}-$ 1.36, $\left.\mathrm{Q}=723.48, \mathrm{I}^{2}=99.66, \mathrm{p}=\leq 0,05\right)$, lo que muestra un efecto grande, lo que indica una reducción de la PIO por lo que señala una estrategia preventiva que se podría de tomar en cuenta. Con respecto al ejercicio contra resistencia el TE fue de 0,13 , lo que indica que no hay efecto. En conclusión, la PIO se reduce por medio del ejercicio aeróbico agudo.

Palabras claves: presión intraocular, actividad física, ejercicio, glaucoma.

\begin{abstract}
High intraocular pressure (IOP) affects visual health; mainly it is a risk factor for glaucoma. The objective of this study was to know the effects of intense exercise on IOP. Methodology: Meta-analyzed experimental studies that evaluated the effect of intense physical activity on intraocular pressure. The research of data encompassed nine multidisciplinary databases. A PRISMA protocol was applied, with criteria of eligibility, methodological quality, and extraction of information by two researchers to guarantee reproducibility. The meta-analysis was based on random effects, heterogeneity with DerSimonian-Lairds. Results: Twenty-nine articles were analyzed to obtain the effect sizes (TE), the experimental groups included 1016 subjects and the control groups 120 subjects. A total of 102 delta TE were calculated. The results of this study show an effect size of
\end{abstract}


0.31 (IC $95 \%=$ de -0.46 a $\left.1.08, Q=16.15, \mathrm{I}^{2}=81.42 \mathrm{p}=\leq 0,05\right)$ for the control group. For the experimental groups an effect of $-0,68$ (IC95\% $=$ de -0.95 a $-0.41, Q=1573.49, I^{2}=99.80$, $\mathrm{p}=\leq 0,05)$. In aerobic exercise, TE was $-1.74(95 \% \mathrm{CI}=-2.11$ to $-1.36, \mathrm{Q}=723.48, \mathrm{I} 2=$ 99.66, $\mathrm{p}=\leq 0.05$ ), which shows a large effect, indicating a reduction of IOP. This result indicates a preventive strategy that could be taken in consideration. In regards to the strength exercise, the TE result was 0.13 , which indicates that there is no effect. In conclusion, IOP is reduced by means of intense aerobic exercise.

Keywords: glaucoma, intraocular pressure, physical activity, exercise.

\section{Introducción}

La salud es uno de los conceptos de bienestar integral más importante en el ser humano, y tiene muchos conceptos específicos como es el caso de la salud visual. A nivel mundial hay 253 millones de personas con discapacidad visual, de las cuales 36 millones presentan una condición de ceguera y 217 millones de baja visión (OMS, 2018). En Costa Rica el 5,85\% de la población tiene discapacidad visual, aunque no se conocen los datos en porcentajes por diagnóstico visual (cataratas, retinosis pigmentaria, glaucoma, entre otros.) constituye la discapacidad con mayor prevalencia, es decir, la que abarca el porcentaje más alto de la población en esa condición. (Instituto Nacional de Estadística y Censos, 2014).

Uno de los aspectos que afecta la salud visual es la presión intraocular alta. La presión intraocular (PIO) es la presión que ejercen los líquidos intraoculares contra la pared del ojo, la cual es necesaria para que este órgano se mantenga distendido. La elevación de presión no solo lleva a la compresión mecánica de la óptica, sino también, a la restricción del suministro de sangre al nervio óptico. Por otro lado, los valores normales de la PIO deben de oscilar entre $11 \mathrm{~mm} \mathrm{Hg}$ y $21 \mathrm{~mm} \mathrm{Hg}$ (Roddy, 2014), estos valores se pueden obtener por medio de una tonometría; este es un método indirecto que se utiliza con mucha frecuencia para conocer los niveles de presión intraocular. Una PIO elevada, es un factor de riesgo modificable que se puede y debe controlar, para prevenir el desarrollo o 
el progreso de enfermedades como el glaucoma (Aspinall et al., 2008; Era, Pärssinen, Kallinen, \& Suominen, 2009; Leighton, 1972; Zhu et al., 2018).

Por otro lado, la actividad física y el ejercicio sin lugar a duda tienen un papel determinante en la calidad vida de las personas (Conte et al., 2009; Esfahani, Gharipour, \& Fesharakinia, 2017; Hong et al., 2014; Huang, 2015; Karabatakis et al., 2004; Kinoshita et al., 2016; Rüfer et al., 2014), no sólo porque mejora parámetros de salud importantes, sino también, como un factor de protección y prevención de enfermedades crónicas (Roddy, 2014).

El ejercicio aeróbico, realizado de manera aguda ha ofrecido como resultado una disminución en la PIO. Estos resultados se han dado tomando en cuenta diferentes aspectos como: intensidad, nivel de actividad de la población, duración; por ejemplo perece que los atletas reducen menos la PIO que las personas activas, y estas a su vez, reducen menos la PIO con respecto a personas sedentarias, así entonces pareciera que las personas sedentarias son las que logran una mayor reducción de la PIO, durante el ejercicio (Harris, Malinovsky, Cantor, Henderson, \& Martin, 1992; Lanigan, Clark, \& Hill, 1989). Con respecto a la intensidad del ejercicio existe controversia ya que algunos autores mencionan que con actividades moderadas se dan mayores reducciones (Hong et al., 2014; Yan et al., 2016), mientras que otros recomiendan intensidades más altas (Ozmerdivenli, Simsek, Bulut, Karacabey, \& Saygin, 2006; Read \& Collins, 2011; Yan et al., 2016; Ahmad 1995).

Por otra parte, se ha encontrado que el ejercicio contra resistencia también ayuda en reducir la PIO, especialmente a intensidades moderadas como al del 60\% de 1RM, no así a intensidades más altas como al $80 \%$ de 1RM. Incluso se ha considerado contraindicado realizar ejercicio contra resistencia a altas intensidades con personas con glaucoma (Conte \& Scarpi, 2014; Vera, García-Ramos, Jiménez, \& Cárdenas, 2017; Vieira, 2006), ya que al 
aumentar la intensidad en la que se realiza el ejercicio, aumenta la PIO especialmente en ejercicios del tren superior, no obstante, ellos afirman la necesidad de realizar más estudios del efecto del ejercicio de contra resistencia sobre la PIO (Ashkenazi, Melamed, \& Blumenthal, 1992; Kozobolis, Detorakis, Konstas, Achtaropoulos, \& Diamandides, 2008; Vera, Jiménez, Redondo, Cárdenas, \& García-Ramos, 2018).

Otra de las prácticas de actividad física reportados es el yoga. En un estudio se demuestra una disminución significativa en la PIO posterior a una sesión de yoga (Dimitrova \& Trenceva, 2017), mientras que otro estudio ha encontrado aumentos en la PIO, después de ciertas posturas de yoga (Jasien, Jonas, de Moraes, \& Ritch, 2015).

En vista de que existe controversia en los resultados de los estudios analizados en cuanto al ejercicio y sus características, se elaboró el siguiente metaanálisis que tuvo como objetivo determinar el efecto agudo del ejercicio sobre la PIO para así establecer cuáles características del ejercicio disminuyen significativamente la presión intraocular.

\section{Metodología}

En la aplicación de la metodología se utilizaron los lineamientos PRISMA (Liberati et al, 2009).

\section{Criterios de Elegibilidad}

Los criterios de inclusión del meta-análisis fueron:

-Investigaciones que reportaran la PIO previo al ejercicio y post ejercicio, de forma aguda, en un mismo grupo de sujetos.

- Los estudios que reportaron promedios y desviaciones estándar,.

- Los realizados sólo con humanos.

- Que los artículos científicos para codificar se encontraran inglés o español. 
- Análisis estadísticos comparativos entre el pretest y postest es decir de carácter intragrupos.

\section{Búsqueda de literatura}

En el proceso de la selección de los artículos del estudio, se llevó a cabo una revisión de literatura en las siguientes bases de datos: SportDiscus con texto completo, psycINFO, Psychology and Behavioral Sciences Collection, Rehabilitation \& Sports Medicine Source, google académico, Research Gate, PubMed. Para llevar a cabo esta búsqueda de referencias se utilizó la siguiente frase boleana: "intraocular pressure AND physical activity OR exercise OR fitness OR sport”. Adicionalmente, se realizó una búsqueda específica de las referencias identificadas en los artículos relevantes. Fué contactado un autor principal de cuatro artículos, el cuál envió por correo electrónico la información completa. La búsqueda se efectúo entre marzo del 2018 y mayo del 2018. No se restringió el rango de la fecha de publicación de los estudios.

\section{Selección de estudios y Codificación de la Información}

Para el presente metaanálisis, se codificó el autor, el año de publicación, el país de procedencia y el $\mathrm{N}$ total de cada estudio. Se categorizaron las variables moderadoras de la siguiente manera:

Características de la Muestra: edad promedio, sexo (femenino, masculino, mixto), actividad física de los sujetos (sedentarios, activos, atletas), medición de ojos (ojo derecho, ojo izquierdo, ambos ojos), condición visual (normal, glaucoma, Miopes).

Características del tratamiento: tipo de ejercicio (aeróbico, contra resistencia, yoga), minutos totales de ejercicio aeróbico, intensidad, porcentaje de frecuencia cardiaca máxima (FCmáx.), porcentaje de 1RM, tiempo de medición post ejercicio, número de 
series, número de repeticiones, número de ejercicios, volumen total obtenido por medio de la fórmula: (número de series* número de repeticiones*número de ejercicio*intensidad).

\section{Calidad de estudios individuales}

La calidad de los estudios se calculó, por medio de la calificación de la validez interna, asignando un punto a la presencia de cada uno de los siguientes aspectos: aleatorización, pretest y postest, tratamiento de ejercicio, grupo control. Con el dato obtenido se hizo una correlación para determinar si los TE estaban influenciados por la calidad del estudio.

\section{Procedimiento del cálculo del tamaño de efecto}

Para analizar los tamaños de efecto (TE) que compararon los cambios entre el pretest y el post-test en los grupos experimentales y controles es decir un TE intragrupos, se siguieron los planteamientos propuestos por Thomas y French (1986). La fórmula utilizada fue «resta entre el promedio del post-test y el promedio del pre-test, dividido entre la desviación estándar del pre-test», o sea, Xpostest- Xpretest / DEpre-test. En la presente revisión un TE negativo significa que reducen la PIO y un TE positivo significa que aumenta la PIO. Con el propósito de no sobreestimar el TE debido al tamaño de la muestra, se aplicó la fórmula para obtener el TE corregido: ${ }^{c=1-\frac{3}{4\left(n_{1}+n_{2}-2\right)-9}}$

La significancia estadística para el TE global calculado se estableció para la situación experimental y para los TE de los niveles de las variables moderadoras, utilizando

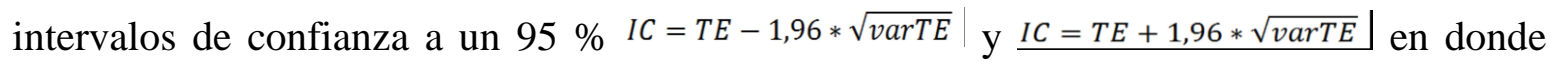
$\operatorname{var}(\mathrm{TE})$ es la varianza de cada tamaño de efecto (Thomas \& French, 1986). Posteriormente, se realizó el cálculo sugerido por Orwin (1983), con el cual se obtiene la cantidad de tamaños de efecto no significativos necesarios para reducir el TE global obtenido a un tamaño de efecto pequeño. Por último, la prueba de homogeneidad $Q$ de Cochran’s 


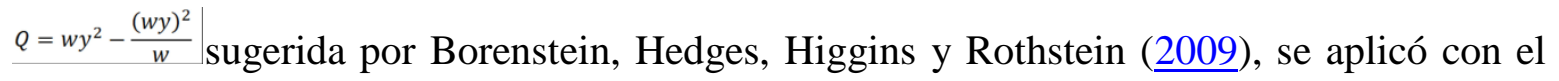
fin de determinar la heterogeneidad de los TE.

\begin{abstract}
Análisis estadístico
Para determinar las diferencias entre los TE de las variables moderadoras discretas se realizaron anovas de una vía, para grupos independientes y se aplicó la técnica de correlación de Pearson para determinar la relación de los TE en las variables moderadoras continuas. Para dichos cálculos se utilizó el programa estadístico IBM-SPSS versión 24® y el programa de hoja de cálculo de Microsoft Excel ${ }^{\circledR}$.

\section{Resultados}

29 estudios fueron codificados (marcados con un * en las referencias) (figura 1) estos generaron 102 tamaños de efecto de los cuales 4 son de grupos control y 98 de grupos experimentales, para un total de 1016 sujetos (133 mujeres, 420 hombres, 463 mixto). Asimismo los estudios incluidos van desde el año 1970 hasta el 2018 y fueron escritos en inglés. En la Tabla 1 se presenta la información de los estudios codificados.
\end{abstract}

A continuación se muestra un diagrama de flujo del proceso de selcción de los artículos científicos analizados. 
Se identificaron 535 publicaciones en la búsqueda inicial en 8 bases de datos: EBSCOHost (incluyó: Academic Search Ultimate, SportDiscus, psycINFO, Psychology and Behavioral Sciences Collection, Rehabilitation \& Sports Medicine Source) google académico, Research Gate, PubMed.

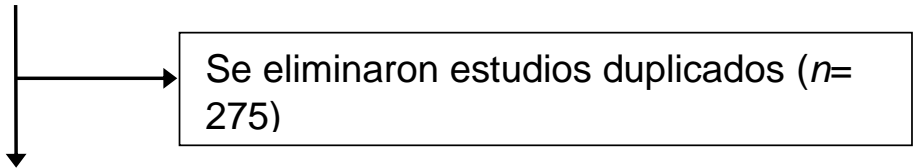

Se revisaron 260 publicaciones en base al título y resumen

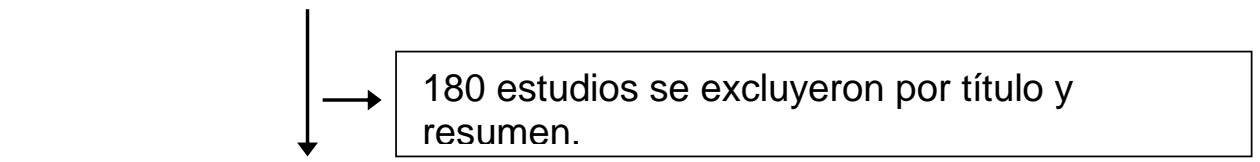

80 publicaciones se evaluaron, con base al texto. 50 no cumplieron con los criterios de elegibilidad.

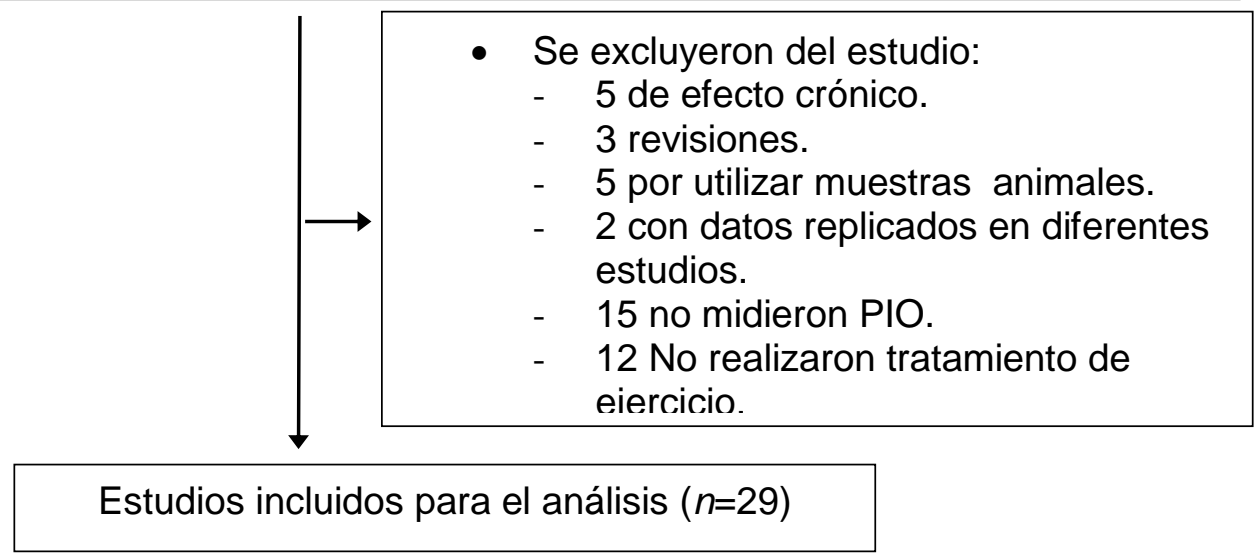

Figura 1. Diagrama de flujo del proceso de selección de los artículos.

En la figura 2, se muestra la correlación de la calidad de los estudios con TE que dio como resultado $\mathrm{r}=0.155$, y una $\mathrm{p}=0.10$, indicando que la calidad de los estudios no afecta los resultados. 


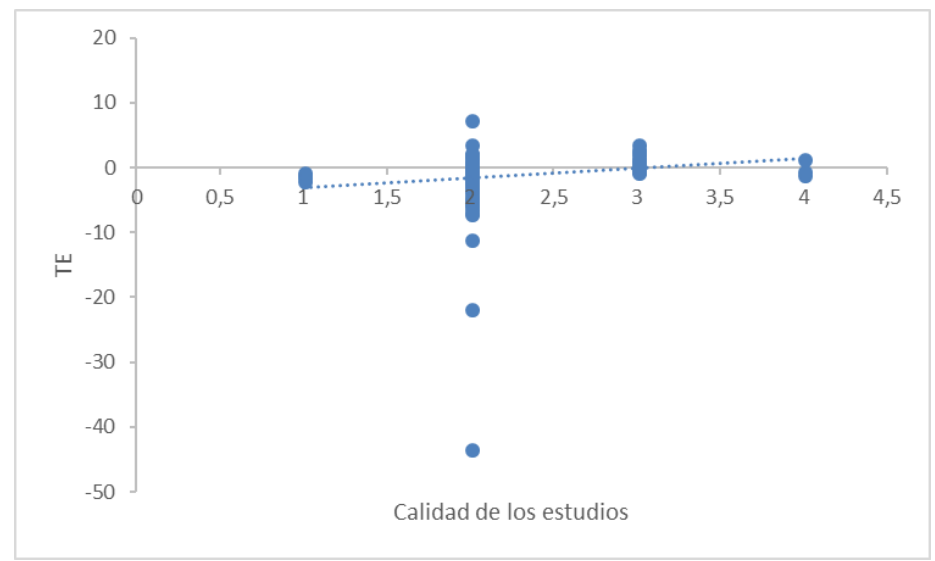

Figura 2. Relación entre la calidad de los estudios y TE. $r=0.155(p=0.10)$.

En la figura 3 se presenta el gráfico de embudo. La prueba de Egger dio como resultado t=-2,09 $(p=0,038)$ indicando que hay asimetría en los estudios, probablemente explicado por la falta de control en las investigaciones analizadas.

Figura 3. Gráfico de embudo.

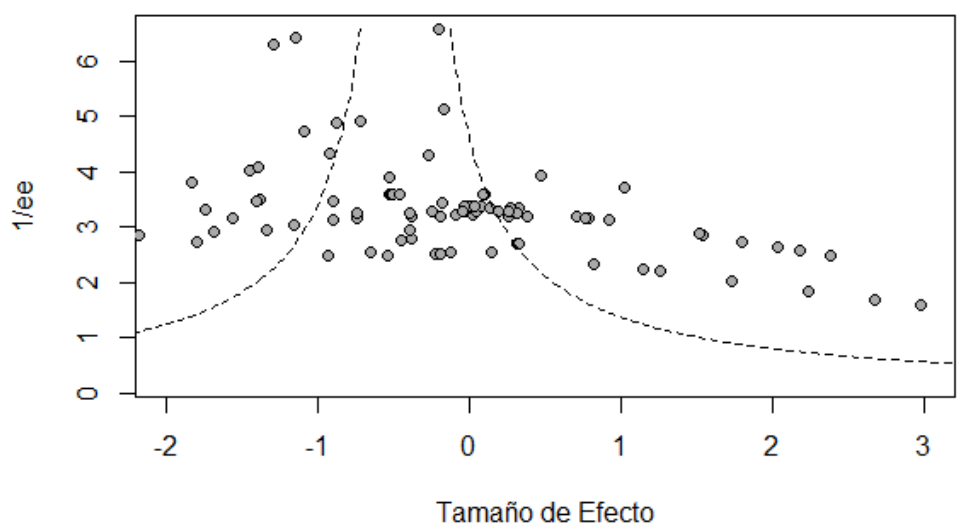

En las figuras 4 y 5 se presentan los forest plots del ejercicio aeróbico y contraresistencia respectivamente, mostrando la estadística descriptiva del TE de cada uno de los estudios. 
Figura 4. Forest Plots de los datos del ejercicio aeróbico

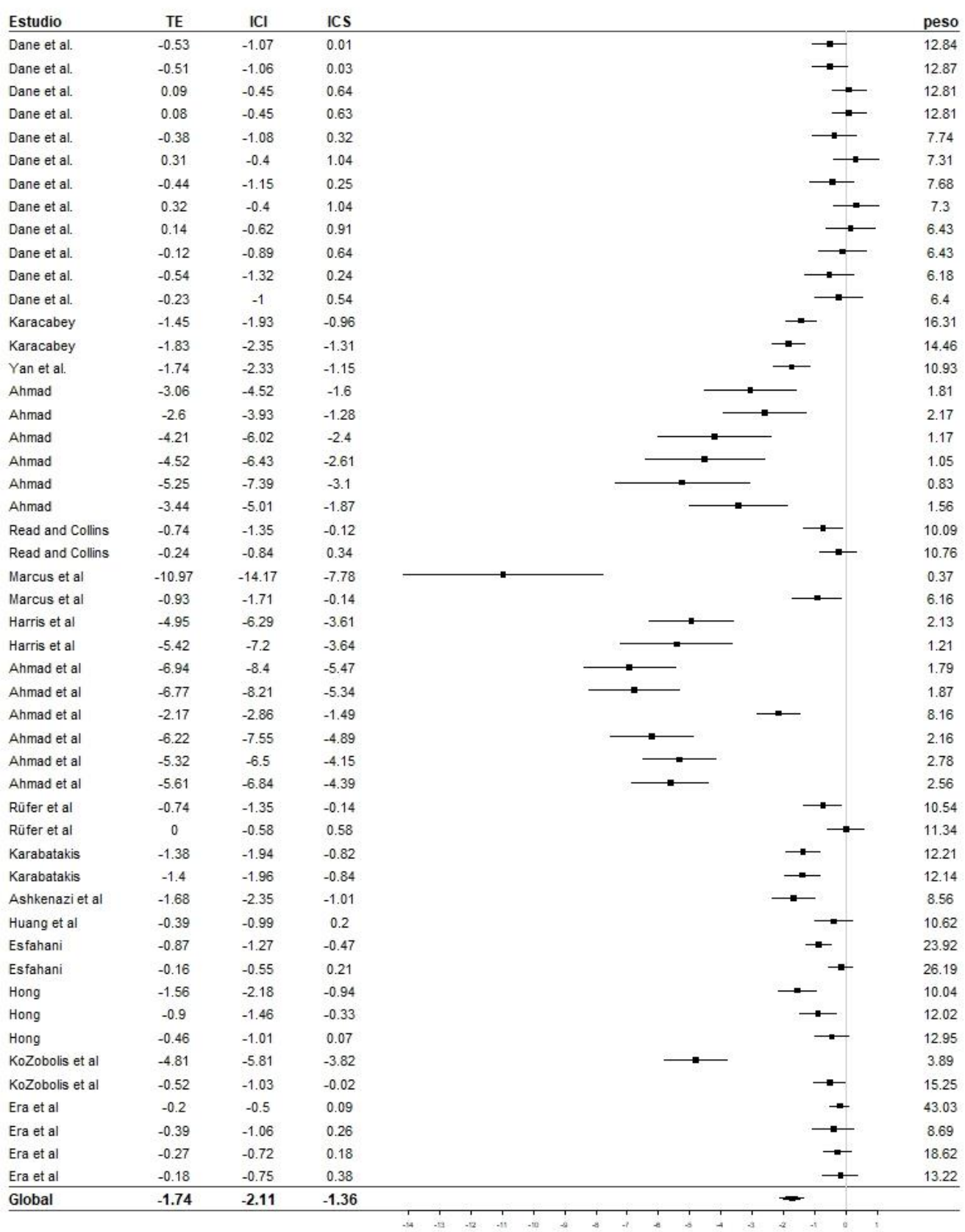


Figura 5. Forest Plots de los datos del ejercicio contra resistencia

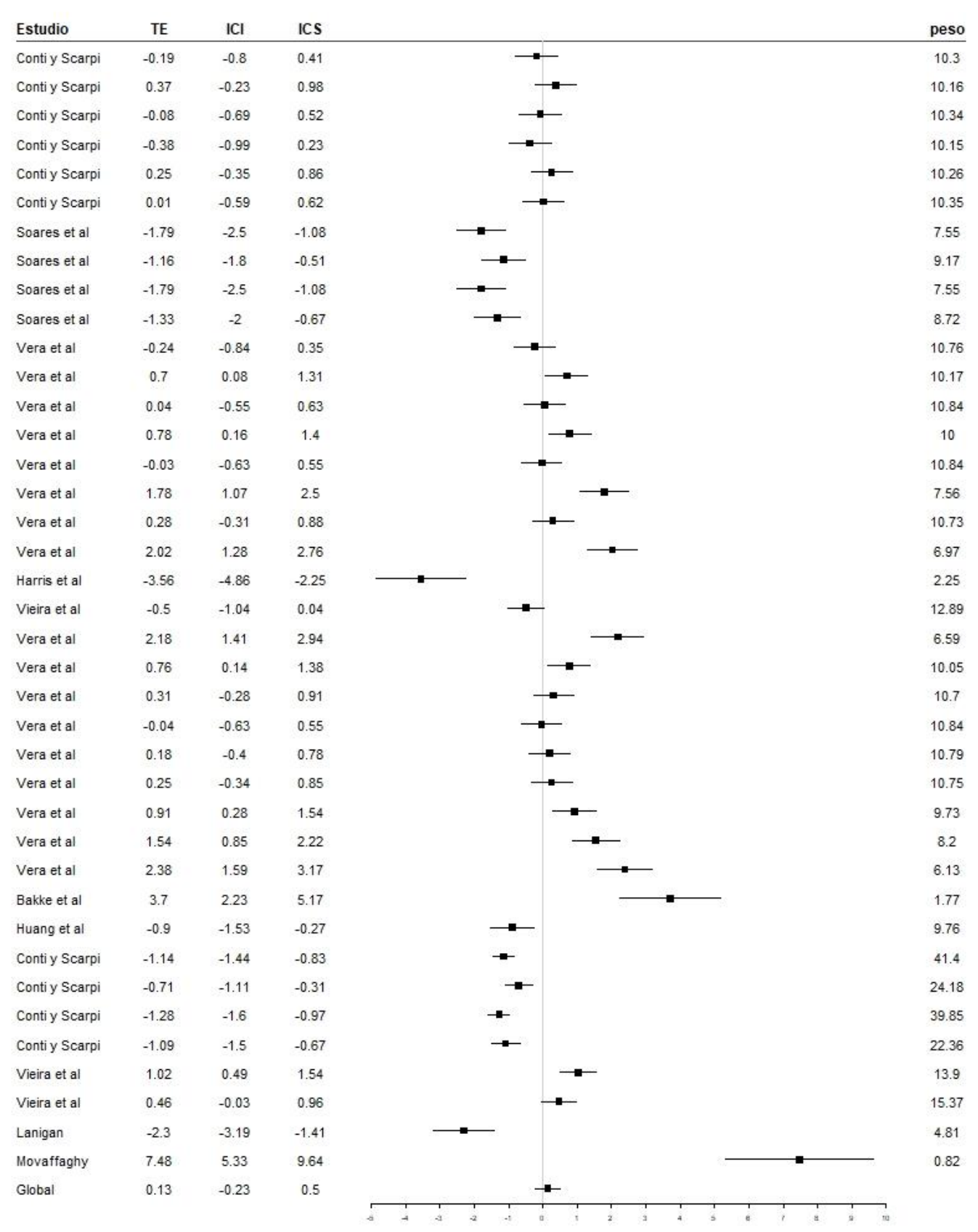


Para cada nivel de las variables moderadoras se obtuvo un intervalo de confianza y de esa forma se estableció la significancia del TE promedio en cada nivel. Para determinar el efecto de las variables moderadoras se realizaron análisis de varianza (ANOVA) (Tabla 1) en las variables discretas.

Tabla 1. Tamaños de efectos calculados

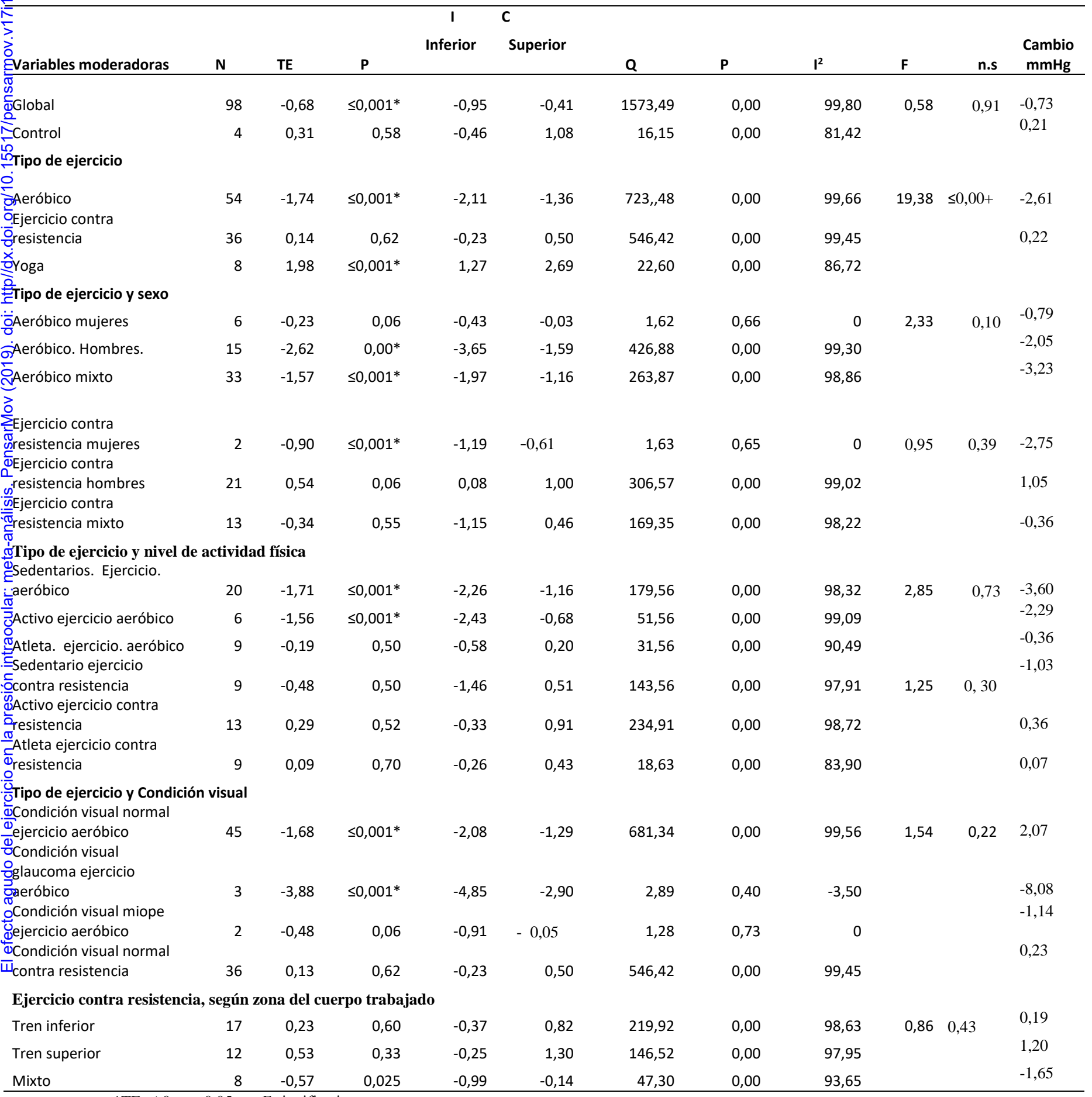

$* \mathrm{TE}: \neq 0, \mathrm{p} \leq 0,05 .+:$ F significativa. 
En la figura 6 se muestra el tamaño de efecto global del grupo experimental y el tamaño de efecto del grupo control, el TE global es de -0,68 el cual es significativamente diferente de cero (intervalo de confianza-0,95 a -0,41). No se encontraron diferencias significativas entre el grupo experimental y el grupo control.

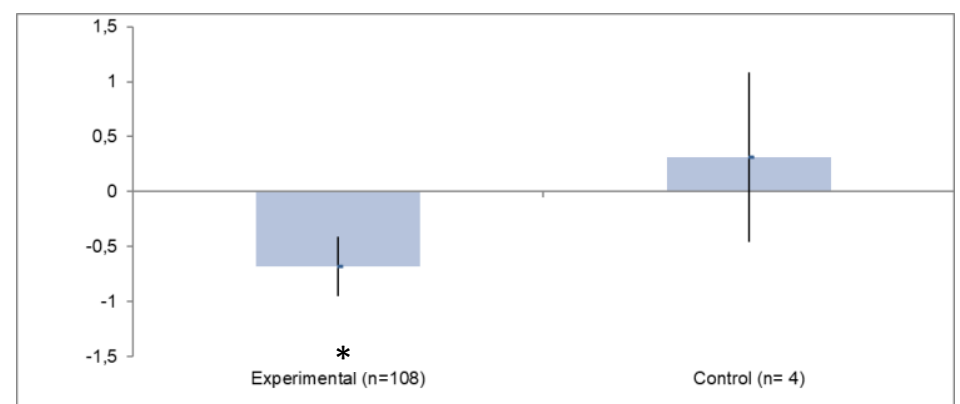

Figura 6. Tamaño de Efecto global para las condiciones experimentales (de -0,68). y grupos controles $(0,30) . * \mathrm{TE}: \neq 0$.

En la figura 7 se muestra el TE en el ejercicio aeróbico según sexo, las mujeres presentaron un TE de $-0,23$, los hombres de $-2,62$ diferente de cero (intervalo de confianza de $-3,65$ a $-0,03$ ) y mixto de $-1,57$ diferente de cero (intervalo de confianza de $-1,97$ a -1,16). No se presentan diferencias significativas entre los grupos.

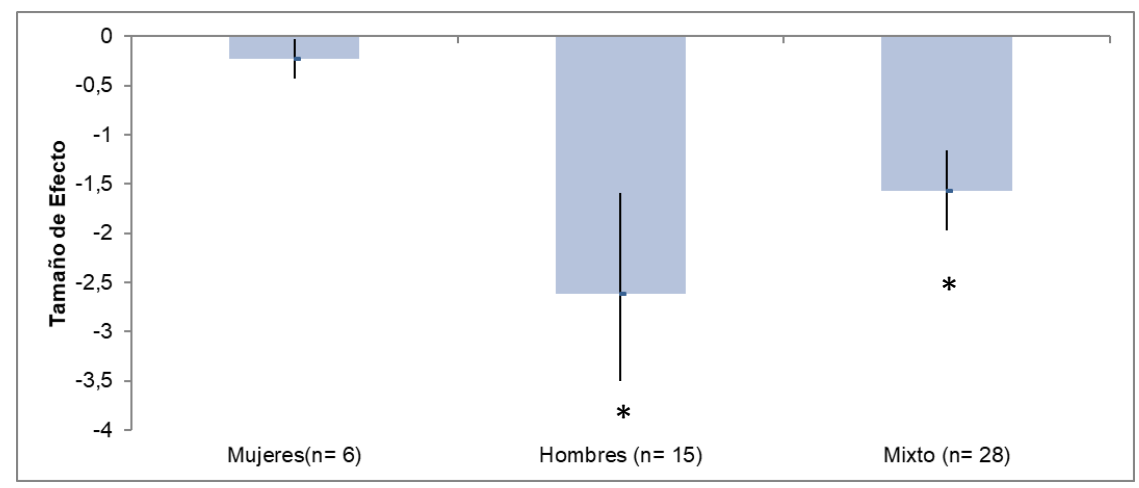

Figura 7. Tamaño de efecto en el ejercicio aeróbico según el sexo. *TE: $\neq 0$ 
En la figura 8 se presentan los resultados de TE en el ejercicio de contra resistencia según sexo, las mujeres presentaron un TE de $-0,90$ diferente de cero (intervalo de confianza de 1,18 a -0,60), los hombres de 0,54 (intervalo de confianza de -0,08 a -1) y mixto de $-0,34$ (intervalo de confianza de $-1,15$ a $-0,46$. No hay diferencias de TE, tampoco diferencias significativas entre los grupos.

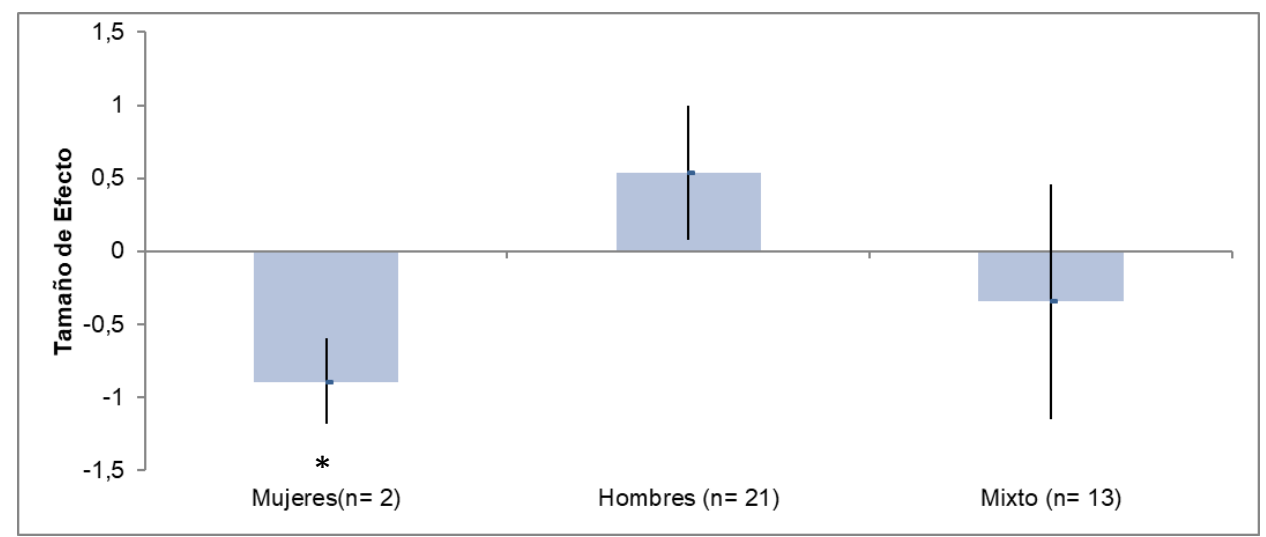

Figura 8. Tamaño de efecto en el ejercicio contra resistencia según el sexo.

En la figura 9 se muestran los TE según nivel de actividad física después de realizar ejercicio aeróbico, los sedentarios tienen un TE de -1,71 diferente de cero (intervalo de confianza de -2,26 a -1,16), los activos un TE de -1,56 diferente de cero (con un intervalo de confianza de -2,43 a -0,68) y las atletas un TE de -0,19 (con un intervalo de confianza de $-0,58$ a $-0,20)$ no presentaron cambios. No se encontraron diferencias significativas entre los grupos.

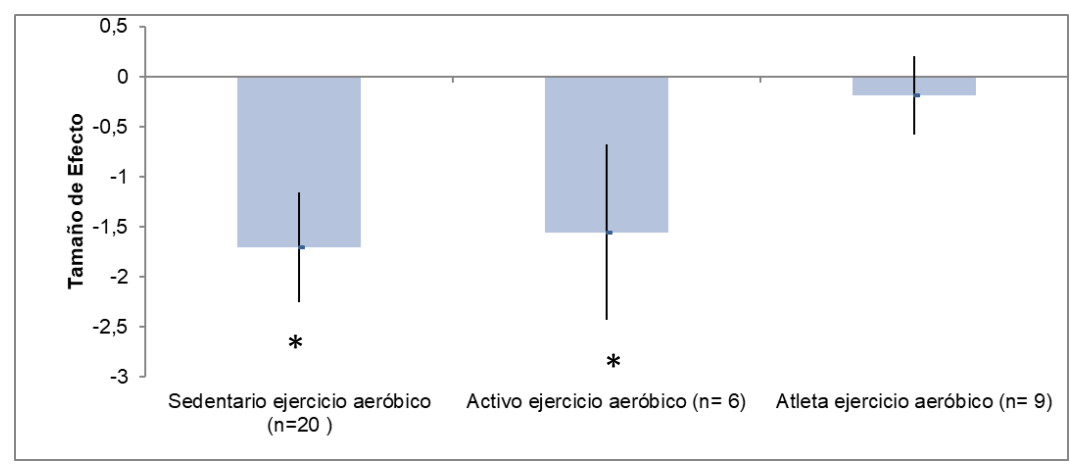


Figura 9. Tamaño de efecto según el nivel de actividad física, después de hacer ejercicio aeróbico. *TE: $\neq 0$

En la figura 10 se muestran los TE según nivel de actividad física después de realizar ejercicio contra resistencia, no se encontraron diferencias de cero. Tampoco se encontraron diferencias significativas entre los grupos.

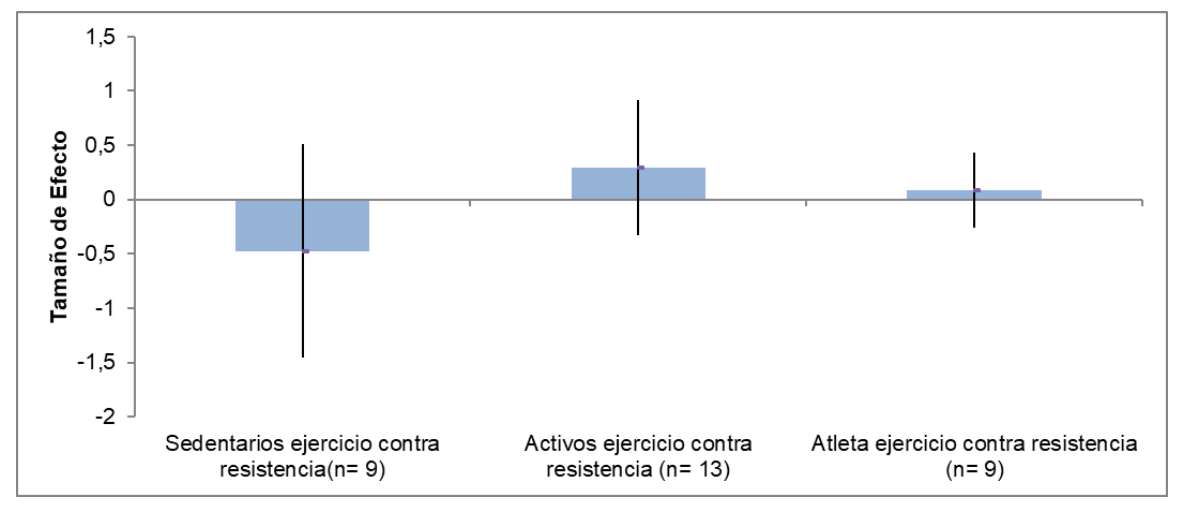

Figura 10. Tamaño de efecto según el nivel de actividad física, después de hacer ejercicio contra resistencia.

En la figura 11 se muestran los TE según la condición visual y el tipo de ejercicio, la condición visión normal y ejercicio aeróbico presentaron un TE de -1,68 diferente de 0 (intervalos de confianza de -2,08 a -1,29), la condición de glaucoma y ejercicio aeróbico de $-3,88$ diferente de 0 (intervalos de confianza de $-4,85$ a -2,9). La condición visual miope y ejercicio aeróbico y visión normal no presentaron cambios en TE. Se encontraron diferencias significativas entre la condición visual de glaucoma y visión normal y entre la condición visual glaucoma y miopes. 


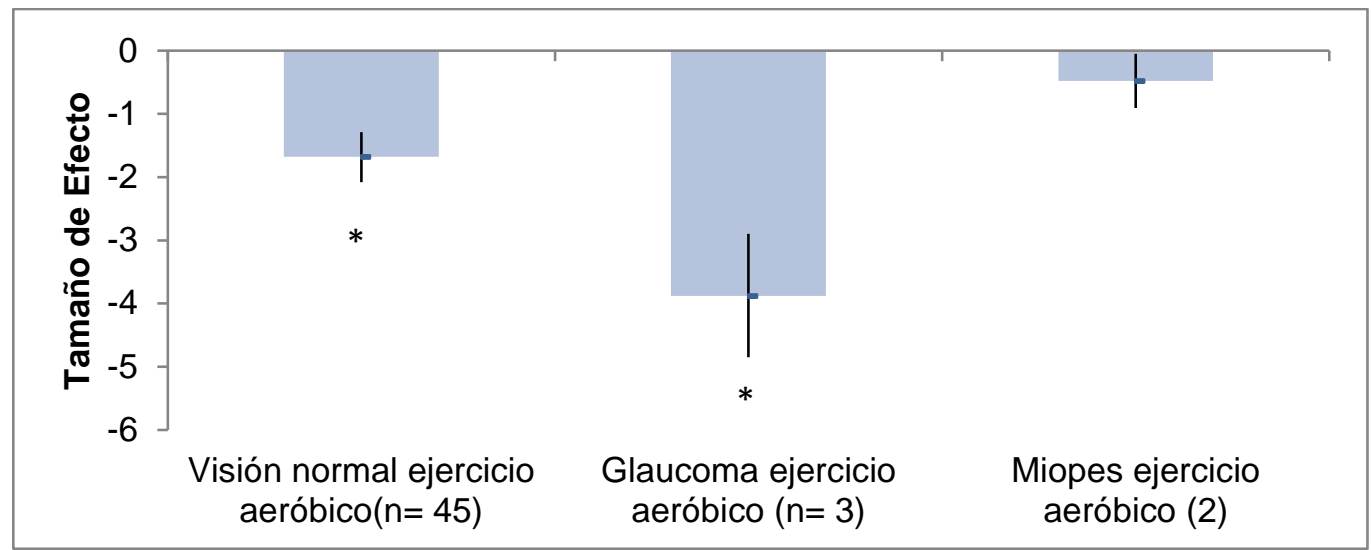

Figura 11. Tamaño de efecto según la condición visual y el tipo de ejercicio. *TE: $\neq 0$

En la figura 12 se muestran los TE del ejercicio contra resistencia según la zona del cuerpo trabajada, no se encontraron diferencias de cero. Tampoco se encontraron diferencias significativas entre los grupos.

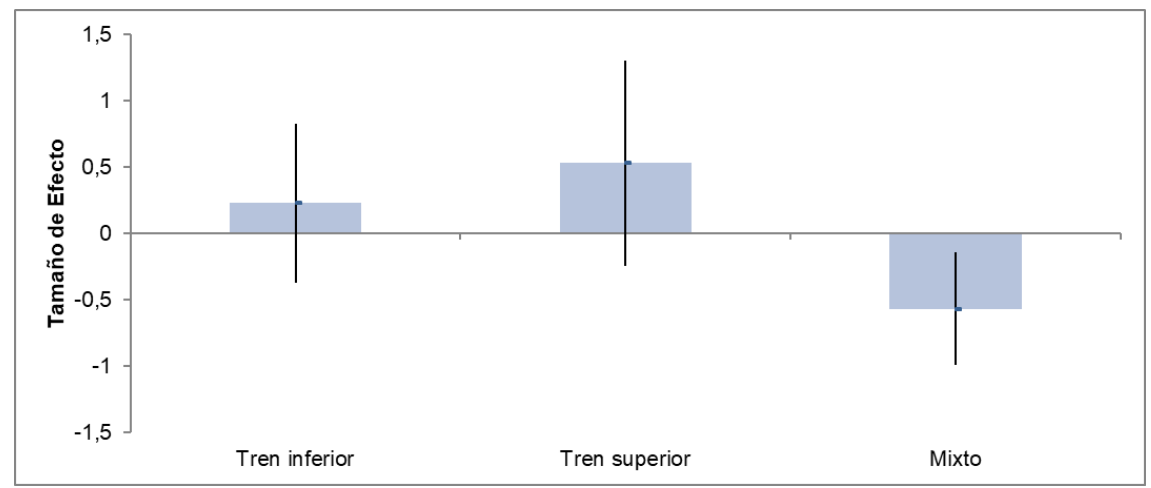

Figura 12. Tamaño de efecto del ejercicio contra resistencia según la zona del cuerpo trabajada. 
En la tabla 2 se presentan los resultados de las variables continuas

Tabla 2. Correlaciones de Pearson entre los TE y las variables continuas

\begin{tabular}{llllr}
\hline & Variables moderadoras & $\mathbf{n}$ & $\mathbf{r}=$ & $\mathbf{p} \leq$ \\
\hline Participantes & Edad & 77 & $-0,176$ & 0,12 \\
Ejercicio aeróbico & Duración de la sesión & 43 & $-0,311$ & $0,042^{*}$ \\
& Intensidad del ejercicio \% FC & 16 & 0,11 & 0,68 \\
& & & & 0,909 \\
Ejercicio contra resistencia & Intensidad del ejercicio \% 1RM & 28 & 0,023 & $0,006^{*}$ \\
& Número de series & 33 & $-0,47$ & $0,00^{*}$ \\
& Número de repeticiones & 28 & $-0,72$ & 0,286 \\
& Número de ejercicio & 35 & $-0,189$ & 0,065
\end{tabular}

$\mathrm{FC}=$ Frecuencia cardiaca, $\mathrm{RM}=$ repetición máxima. $*=\mathrm{p} \leq 0,05$

En la figura 13 se muestra la correlación entre los valores del TE calculado y la duración de la sesión para cada estudio $(\mathrm{n}=43)$, se obtuvo una relación significativa.

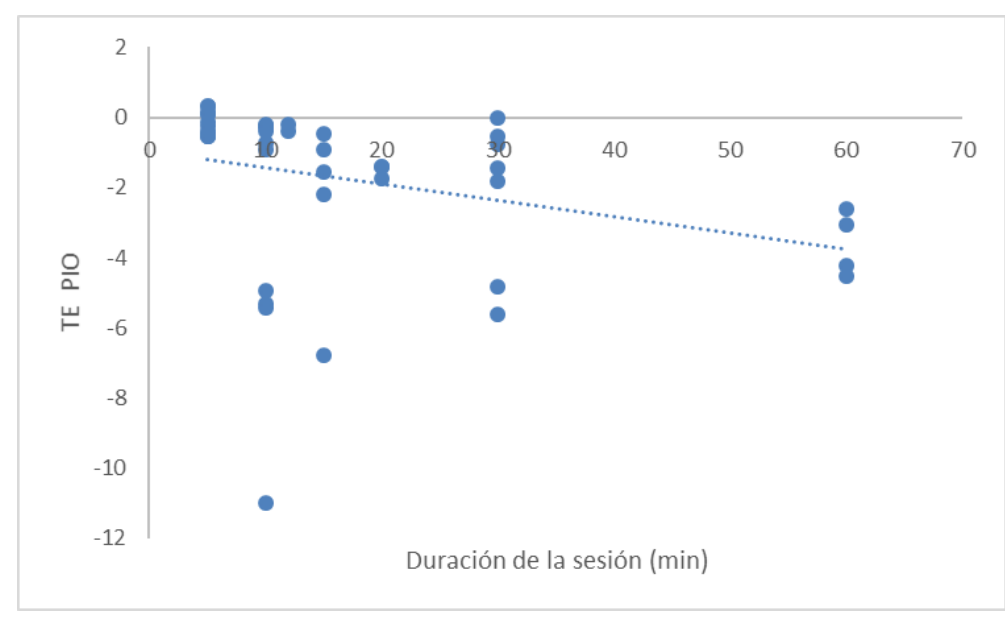

Figura 13. Relación entre el TE y la duración de la sesión. $r=-0,31(\mathrm{p}=0,042)$.

En la figura 14 se muestra la correlación entre los valores del TE calculado y el número de series para cada estudio ( $n=33)$, se obtuvo una relación significativa. 


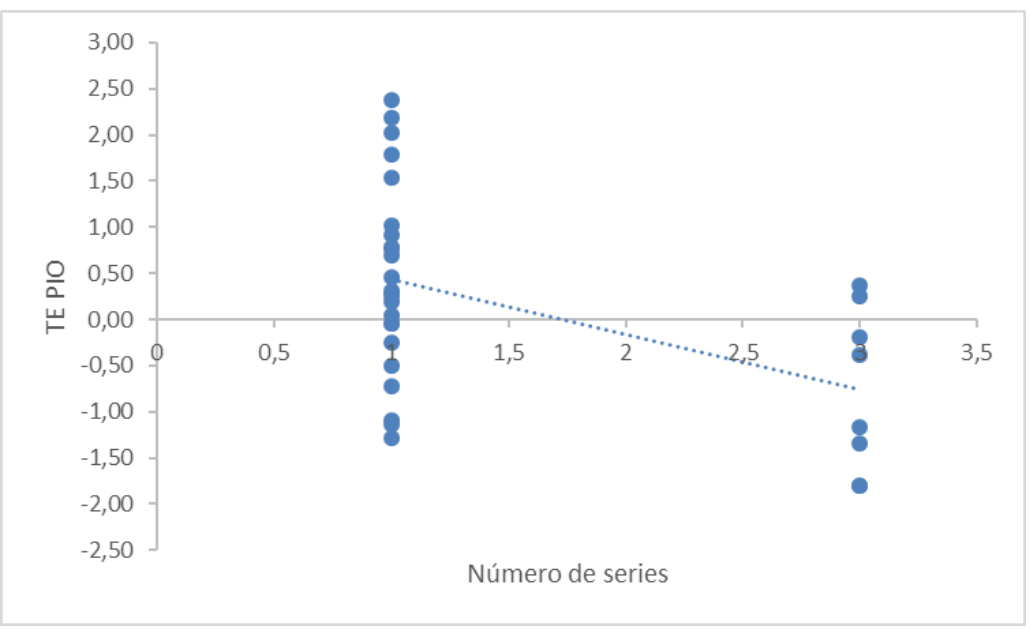

Figura 14. Relación entre el TE y el número de series. $\mathrm{r}=-0,47(\mathrm{p}=0,006)$.

En la figura 15 se muestra la correlación entre los valores del TE calculado y el número de repeticiones para cada estudio ( $\mathrm{n}=28)$, se obtuvo una relación significativa.

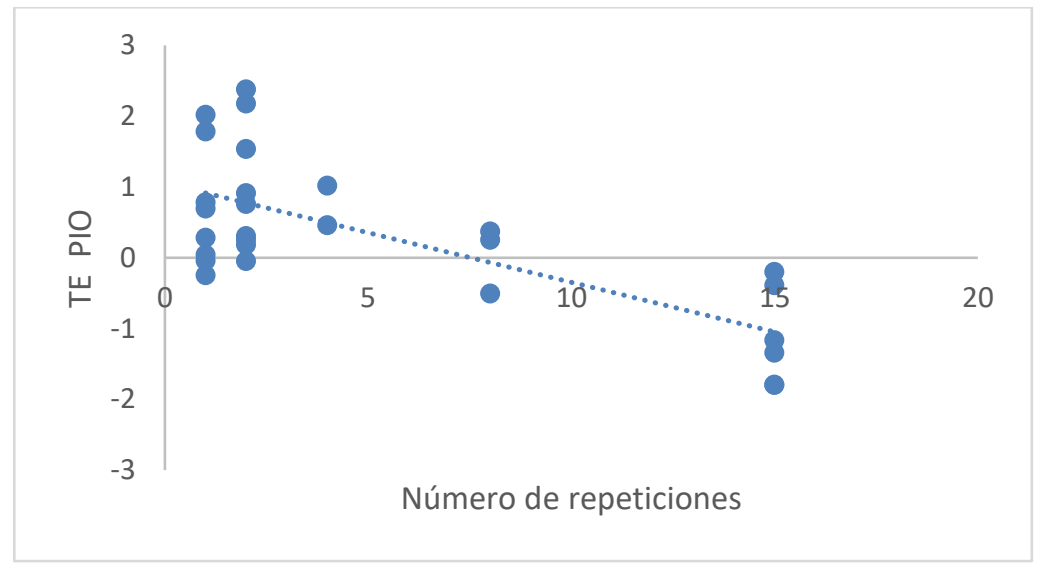

Figura 14. Relación entre TE y el número de repeticiones. $r=-0,72(p=0,00)$.

\section{Discusión}

Los resultados de este estudio muestran un efecto global entre moderado y grande en el grupo experimental (TE= -0.68; n=98), en la PIO después de realizar actividad física de manera aguda, sin embargo no existe grupo control con el cuál comparar. Cabe destacar que un tamaño de efecto de 0.20 representa un tamaño pequeño, 0.50 representa un tamaño 
moderado, mientras que 0.80 representa un tamaño de efecto grande (Cohen,1988). El tamaño global refleja una mejora en la PIO, después de realizar una práctica física de forma aguda, ya que al ser negativo muestra una reducción de esta variable. Por otro lado, en el grupo control $(\mathrm{TE}=0.31 ; \mathrm{n}=4)$, no se encontraron diferencias significativas, pero tampoco los resultados mostraron diferencias considerables con respecto al grupo experimental, probablemente por el "n" tan pequeño. Seguidamente, se prosiguió con la separación entre los tamaños de efecto por tipo de ejercicio para una mejor compresión y análisis.

La magnitud encontrada en el ejercicio aeróbico agudo en la $\mathrm{TE}=-1.74$, lo que demuestra un tamaño de efecto grande. Los estudios de Dane, Koçer, Demirel, Üçok, \& Tan, (2006); Ozmerdivenli et al. (2006); Read \& Collins, (2011), demuestran reducciones de la PIO con los tratamientos efectuados con ejercicio aeróbico agudo. Además, algunas de las probables razones por las que el ejercicio aeróbico podría disminuir la PIO es por la reducción del humor acuoso, este se da porque al perder agua por medio del sudor se incrementa la presión osmótica del plasma y también al aumentar la concentración de sangre en los músculos activos se reduce el flujo sanguíneo ocular. De igual forma, el sistema nervioso simpático causa vasoconstricción coroidea por lo que se reduce el flujo de sangre y disminuya la PIO. Además, al aumentar la concentración de lactato, disminuye el ph en sangre y hay hipercapnia con lo que se logra un mejor drenaje acuoso (Yan et al., $\underline{2016}$.

El análisis efectuado para determinar si el sexo influye en el tipo de ejercicio, demostró TE significativos en el caso del ejercicio aeróbico de los hombres y el mixto, por otro lado la PIO en mujeres no encontró diferencias significativas, es importante tomar en cuenta que solo se encontraron dos estudios, lo que podría explicar este resultado al ser un “n” tan pequeño. Además podría ser que el sexo sea un factor que influya en la PIO (Dane 
et al., 2006; Era et al. 2009), por lo que se recomienda realizar análisis por separado para conocer la tendencia de la PIO según sexo.

Cabe señalar que en los resultados del ejercicio contra resistencia, la TE resulta positiva, en consecuencia la PIO aumenta, sin embargo este incremento no es estadisticamente significativo. Este resultado debe manejarse con cautela ya que en la mayoría de estudios individuales la PIO se incrementa con este tipo de ejercicio (Bakke; Hisdaly \& Semb 2009; Conte \& Scarpi, 2014; Vera et al., 2017; Vieira, 2006).

Con respecto a los ejercicios de yoga, que involucren la inversión de cabeza el resultado de el $\mathrm{TE}=1,98$ lo que indica un incremento significativo de la PIO. Tal es el caso del estudio de Gallardo; Aggarwal; Cavanagh \& Whitson (2006) en donde después de realizar una Sirsasana de yoga (cabeza hacia abajo), el incremento de la PIO fue de $10 \mathrm{~mm}$ Hg. Ellos mencionan, que este tipo de prácticas son de alto riesgo para personas con glaucoma, ya que las posturas en donde las personas están con la cabeza abajo (paradas de manos) o con los pies encima de la cabeza podrían provocar una elevación de la PIO, por un incremento de la vasculatura coroidea y una mayor presión venosa epiescleral, en la cual el flujo de tejidos oculares se compromete, y la coroides es un tejido muy vascularizado que puede causar cambios dinámicos en el líquido del humor acuoso (Soares et al., 2015) .

El TE en personas sedentarias muestra un resultado significativo de -1.71 , y en personas activas de -1.56 , en ambos casos diferente de cero, por otro lado en las personas atletas el TE fue de -0,19. Estos tamaños de efecto están acorde a la literatura, en donde el nivel de actividad física influye sobre las reducciones de la PIO, las personas sedentarias presentan las reducciones más grandes, después las personas activas y los atletas no 
presentaron cambios (Dane et al., 2006; Harris et al., 1994; Ozmerdivenli et al., 2006). Estos resultados ameritan más análisis para determinar las posibles causas.

Con respecto a la condición visual y el ejercicio aeróbico, los TE son significativos en la condición visual normal $(-1,68)$ y en la condición de glaucoma $(-3,88)$. Estos datos son muy importantes porque demuestran que el ejercicio aeróbico beneficia la disminución de la PIO, con lo que se puede obtener beneficios significativos especialmente en las personas que sufren de glaucoma, esto brinda una recomendación importante, ya que se debería incorporar como parte del tratamiento la práctica regular de actividad física para así poder reducir la PIO, y mejorar su salud visual (Ahmad, 1995). Además Leske y Podgor (1983), mencionan que cuando una persona reduce $1 \mathrm{mmHg}$, disminuye en un $10 \%$ la tasa de progreso de la enfermedad y al aumentar $1 \mathrm{mmHg}$, incrementa en un $19 \%$.

Con respecto a la duración del ejercicio, se encuentra una correlación significativa, lo que indica que a mayor duración el ejercicio mayor disminución de la de la PIO. Este resultado estimula y enfatiza el tiempo como una variable de peso a tomar en cuenta en investigaciones con esta línea, cabe señalar que en ninguna investigación se tomó como variable de estudio.

Por otro lado, tanto el número de series como el número de repeticiones correlacionan significativamente en la disminución de la PIO. En ambos casos a mayor número de series y o repeticiones mayor disminución de la PIO. Al aumentar el número de series y el número de repeticiones en los ejercicios de contra resistencia, estos se deben hacer a menor intensidad, es decir que se orienten más a la resistencia por lo que no hay tanto compromiso de apnea voluntaria (suspender respiración), ya que disminuye el retorno venoso, lo que perjudica el drenaje del humor acuoso o bien tampoco hay tanta presión en el tórax y el abdomen (maniobra de Valsalva) (Soares et al., 2015). 


\section{Conclusiones y recomendaciones}

La conclusión más importante es que el ejercicio físico realizado de forma aguda ejerce un efecto positivo en la PIO; es decir que al realizar ejercicio la presión intraocular disminuye.

Así mismo el efecto del ejercicio se da tanto en personas con condición visual normal y especialmente tiene una magnitud de TE grande en personas con glaucoma, lo que da pie a que parte de su tratamiento podría ser la práctica de ejercicio y actividad física y así mejoren su salud visual.

Del mismo modo el ejercicio mejora la PIO en personas activas y sedentarias, lo que indica que la condición física es un elemento que influye en la reducción de la PIO.

Si bien es cierto que a mayor duración de ejercicio mayor reducción de PIO, también con duraciones cortas se han encontrado efectos positivos.

Se necesitan investigaciones fuertes en diseños experimentales, es decir que tengan

grupo control, que se aleatorice y que estudien diferentes variables como el sexo, la edad, no solo como estadística descriptiva sino como parte del estudio.

Se recomienda realizar estudios de yoga para conocer su efecto, pero que no incluyan inversiones de cabeza.

En el área de contra resistencia se evidencia que existen pocos estudios y débiles diseños de investigación por lo que sería un área a desarrollar.

\section{Fortalezas, limitaciones e implicaciones}

Este metaanálisis es el primero que muestra estas variables moderadoras en ejercicio aeróbico y en ejercicio de contra resistencia. 
Se muestran resultados interesantes que podrían dar pie a siguientes estudios como por ejemplo que se compare la duración del ejercicio y su efecto en la PIO. Así como realizar estudios que comparen los resultados entre hombres y mujeres.

Por último se recomienda a los investigadores de esta área reportar la estadística descriptiva para que los estudios puedan ser meta analizados.

\section{Referencias}

Las referencias marcadas con un asterisco indican estudios comprendidos en el meta-análisis.

*Ahmad, I (1995). The effects of Mild, moderate and severe Exercise on intraocular Pressure in Glaucoma Patients. Japanese Journal of Physiology. 45(4), 561-569. doi https://doi.org/10.2170/jjphysiol.45.561

*Ashkenazi, I., Melamed, S., \& Blumenthal, M. (1992). The effect of continuous strenuous exercise on intraocular pressure. Investigative ophthalmology \& visual science, 33(10), 2874-7. Recuperado de https://www.ncbi.nlm.nih.gov/pubmed/1526737

Aspinall, P. A., Johnson, Z. K., Azuara-Blanco, A., Montarzino, A., Brice, R., \& Vickers, A. (2008). Evaluation of Quality of Life and Priorities of Patients with Glaucoma. Investigative Opthalmology \& Visual Science, 49(5), 1907-1915. doi:10.1167/iovs.07-0559

*Bakke, E. F., Hisdal, J., \& Semb, S. O. (2009). Intraocular Pressure Increases in Parallel with Systemic Blood Pressure during Isometric Exercise. Investigative Opthalmology \& Visual Science, 50(2), 760. doi:10.1167/iovs.08-2508

Borenstein, M., Hedges, L.H., Higgins, J.P.T. \& Rothstein, H.R. (2009). Introduction to Meta-Analysis. Wiltshire, Inglaterra: Wiley. Recuperado de http://www.wiley.com/WileyCDA/WileyTitle/productCd-EHEP002313.html 
Organización Mundial de la Salud. (20 de junio de 2018). Ceguera y discapacidad visual. Recuperado de http://www.who.int/es/news-room/fact-sheets/detail/blindness-andvisual-impairment

*Conte, M., \& Scarpi, M. J. (2014). A comparison of the intraocular pressure response between two different intensities and volumes of resistance training. Revista Brasileira de Oftalmologia, 73(1). doi:10.5935/0034-7280.20140005

*Conte, M., Scarpi, M. J., Rossin, R. A., Beteli, H. R., Lopes, R. G., \& Marcos, H. L. (2009). Variação da pressão intraocular após teste submáximo de força no treinamento resistido. Arquivos Brasileiros de Oftalmologia, 72(3), 351-354. doi:10.1590/S0004-27492009000300013

*Dane, S., Koçer, I., Demirel, H., Üçok, K., \& Tan, Ü. (2006). Effect of acute submaximal exercise on intraocular pressure in athletes and sedentary subjets. International Journal of Neuroscience, 116(10), 1207-1214. doi: https://doi.org/10.1080/00207450500522501

*Dimitrova, G., \& Trenceva, A. (2017). The short-term effect of yoga ocular exercise on intra-ocular pressure. Acta Ophthalmologica, 95(1), e81-e82. doi:10.1111/aos.12850

Era, P., Pärssinen, O., Kallinen, M., \& Suominen, H. (2009). Effect of bicycle ergometer test on intraocular pressure in elderly athletes and controls. Acta Ophthalmologica, 71(3), 301-307. doi:10.1111/j.1755-3768.1993.tb07139.x

*Esfahani, M., Gharipour, M., \& Fesharakinia, H. (2017). Changes in intraocular pressure after exercise test. Oman Journal of Ophthalmology, 10(1), 17. doi:10.4103/0974620X.200689 
*Gallardo, M. J., Aggarwal, N., Cavanagh, H. D., \& Whitson, J. T. (2006). Progression of glaucoma associated with the Sirsasana (headstand) yoga posture. Advances in Therapy, 23(6), 921-925. doi:10.1007/BF02850214

*Harris, A., Malinovsky, V. E., Cantor, L. B., Henderson, P. A., \& Martin, B. J. (1992). IsocQpniQ Blocks Exercise-Induced Reductions in Ocular Tension, 33, 4.

*Harris, A., Malinovsky, V., \& MartinX, B. (1994). Correlates of acute exercise-induced ocular hypotension., 35(11), 6 .

*Hong, J., Zhang, H., Kuo, D. S., Wang, H., Huo, Y., Yang, D., \& Wang, N. (2014). The Short-Term Effects of Exercise on Intraocular Pressure, Choroidal Thickness and Axial Length. PLoS ONE, 9(8), 1-6. doi:10.1371/journal.pone.0104294

*Huang, R. (2015). The Effect of Dynamic and Isometric Exercise on Refractive State, Accommodation and Intra-Ocular Pressure. Advances in Ophthalmology \& Visual System, 2(3). doi:10.15406/aovs.2015.02.00047

Instituto Nacional de Estadística y Censos. (Ed.). (2014). Costa Rica a la luz del Censo del 2011. San José, Costa Rica: Instituto Nacional de Estadística y Censos.

*Jasien, J. V., Jonas, J. B., de Moraes, C. G., \& Ritch, R. (2015). Intraocular Pressure Rise in Subjects with and without Glaucoma during Four Common Yoga Positions. PLOS ONE, 10(12), 1-16. doi:10.1371/journal.pone.0144505

*Karabatakis, V. E., Natsis, K. I., Chatzi balis, T. E., Lake, S. L., Bisbas, I. T., Kallinderis, K. A., \& Stangos, N. T. (2004). Correlating Intraocular Pressure, Blood Pressure, and Heart Rate Changes after Jogging. European Journal of Ophthalmology, 14(2), 117-122. doi:10.1177/112067210401400206 
Kinoshita, T., Mori, J., Okuda, N., Imaizumi, H., Iwasaki, M., Shimizu, M., .. Mitamura, Y. (2016). Effects of Exercise on the Structure and Circulation of Choroid in Normal Eyes. PLOS ONE, 11(12), 1-14. doi:10.1371/journal.pone.0168336

*Kozobolis, V. P., Detorakis, E. T., Konstas, A. G., Achtaropoulos, A. K., \& Diamandides, E. D. (2008). Retrobulbar blood flow and ophthalmic perfusion in maximum dynamic exercise. Clinical \& Experimental Ophthalmology, 36(2), 123-129. doi:10.1111/j.1442-9071.2007.01646.x

*Lanigan, L. P., Clark, C. V., \& Hill, D. W. (1989). Intraocular pressure responses to systemic autonomic stimulation. Eye, 3(4), 477-483. doi:10.1038/eye.1989.72

Leske, C and Podgor, M (1983). Indidence estimates for lens changes, macular changes, open-angle glaucoma and diabetic retinopathy. American Journal of Epidemmiology, 118(2), 206-212. doi:10.1093/oxfordjournals.aje.a113628

Leighton, D. A. (1972). Effect of walking on the ocular tension in open-angle glaucoma. British Journal of Ophthalmology, 56(2), 126-130. doi:10.1136/bjo.56.2.126

Orwin, R. G. (1983). A fail-safe $\mathrm{N}$ for effect size in meta-analysis. Journal of Educational Statistics, 8(2),157-159. Recuperado de http://www.jstor.org/stable/1164923?seq=1

*Ozmerdivenli, R., Simsek, E., Bulut, S., Karacabey, K., \& Saygin, O. (2006). Comparison of the effects of acute an regular exercise on intraocular pressure in turkish athlete and sedentarians. International Journal of Neuroscience, 116(3), 351-360. doi:10.1080/00207450500442288

*Read, S. A., \& Collins, M. J. (2011). The short-term influence of exercise on axial length and intraocular pressure. Eye, 25(6), 767-774. https://doi.org/10.1038/eye.2011.54

Roddy, G., Curnier, D., \& Ellemberg, D. (2014). Reductions in Intraocular Pressure After Acute Aerobic Exercise: A Meta-Analysis. Clinical Journal Sport Medicine, 24(5), 364-372. 
*Rüfer, F., Schiller, J., Klettner, A., Lanzl, I., Roider, J., \& Weisser, B. (2014). Comparison of the influence of aerobic and resistance exercise of the upper and lower limb on intraocular pressure. Acta Ophthalmologica, 92(3), 249-252. doi:10.1111/aos.12051

*Soares, A. S., Caldara, A. A., Storti, L. R., Teixeira, L. F. M., Terzariol, J. G. T., \& Conte, M. (2015). Variation of intraocular pressure in resistance exercise performed in two different positions. Revista Brasileira de Oftalmologia, 74(3). doi.org:10.5935/0034-7280.20150033

Thomas, J., \& French, K. (1986). The Use of Meta-Analysis in Exercise and Sport: A Tutorial. Research Quarterly for Exercise and Sport, 57(3), 196-204. doi: https://doi.org/10.1080/02701367.1986.10605397

*Vera, J., García-Ramos, A., Jiménez, R., \& Cárdenas, D. (2017). The acute effect of strength exercises at different intensities on intraocular pressure. Graefe's Archive for Clinical and Experimental Ophthalmology, 255(11), 2211-2217. doi:10.1007/s00417-017-3735-5

*Vera, J., Jiménez, R., Redondo, B., Cárdenas, D., \& García-Ramos, A. (2018). Fitness Level Modulates Intraocular Pressure Responses to Strength Exercises. Current Eye Research, 1-7. doi:10.1080/02713683.2018.1431289

*Vieira, G. M. (2006). Intraocular Pressure Variation During Weight Lifting. Archives of Ophthalmology, 124(9), 1251. doi:10.1001/archopht.124.9.1251

*Yan, X., Li, M., Song, Y., Guo, J., Zhao, Y., Chen, W., \& Zhang, H. (2016). Influence of Exercise on Intraocular Pressure, Schlemm's Canal, and the Trabecular Meshwork. Investigative Opthalmology \& Visual Science, 57(11), 4733. doi:10.1167/iovs.1619475 
Zhu, M., Lai, J., Choy, B., Shum, J., Lo, A., Ng, A., Cheuk, J., \& So, K. (2018). Physical exercise and glaucoma: a review on the roles of physical exercise on intraocular pressure control, ocular blood flow regulation, neuroprotection and glaucomarelated mental health. Acta Ophthalmologica, 96(6), e676-e691.Recuperado de https://www.ncbi.nlm.nih.gov/pubmed/29338126 